\title{
Bayesian Reasoning and Artificial Intelligence
}

\author{
MICHAEL GR. VOSKOGLOU \\ Mathematical Sciences, School of Technological Applications \\ University of Peloponnesus (ex T. E. I. of Western Greece \\ Meg. Alexandrou 1 - 26334 Patras \\ GREECE
}

\begin{abstract}
The present article studies the importance of Bayesian Reasoning in everyday life situations and for the whole science. Examples are also given to illustrate our results.
\end{abstract}

Key-Words: - Bayes' Theorem, Bayesian Reasoning, Artificial Intelligence (AI), Scientific Method, Trial and Error.

Received: March 3, 2020. Revised: August 16, 2020. Accepted: August 31, 2020.

Published: September 10, 2020.

\section{Introduction}

Artificial Intelligence (AI) is the branch of Computer Science that focuses on the theory and practice of creating intelligent machines, which work and react like humans. The term AI was first coined by John McCarthy in 1956, when he held the first academic conference on the subject. in Dartmouth college, USA [1]. However, the journey to understand if machines can truly think began much earlier; e.g. Alan Turin's universal machine in 1936 [2].

AI has roots in mathematics, engineering, technology and science and as a synthesis of ideas from all those fields has created a new situation that is only just beginning to generate enormous changes and benefits to the human society.

Probability theory is one of the main mathematical tools used in AI applications. Edwin T. Jaynes (1922-1998), Professor of Physics at the University of Washington, was the first who argued that Probability theory could be considered as a generalization of the bivalent logic reducing to it in the special case where our hypothesis is either absolutely true or absolutely false [3]. Many eminent scientists have been inspired by the ideas of Janes, like the expert in Algebraic Geometry David Mumford, who believes that Probability and Statistics are emerging as a better way for building scientific models [4].

Nevertheless, both Probability and Statistics have been developed on the basis of the principles of the bivalent logic. As a result, they are tackling effectively only the cases of the existing in real world uncertainty due to randomness and not those due to imprecision. In cases of imprecision, the Zadeh's Fuzzy Logic (FL) comes to bridge the existing gap $[5,6]$.

However, as we shall see in the next section, although probabilities have been defined and developed on the basis of principles of the bivalent logic, the Bayesian rule calculating the value of the conditional probabilities, introduces a kind of multi-valued logic tackling the existing due to imprecision uncertainty in a way analogous to fuzzy logic!

The present work focuses on illustrating the importance of Bayesian reasoning to everyday life and science and in extension to AI. The rest of the article is formulated as follows: The Bayes' rule is presented in next section and its importance for $\mathrm{AI}$ is justified. The third section includes applications of this rule to everyday life situations. In fourth section, the argument that the whole science could be considered as a Bayesian process is discussed and the article closes with the general conclusion presented in section five.

\section{The Bayes' Theorem}

Let $\mathrm{A}$ and $\mathrm{B}$ be two intersecting events. Then it is straightforward to check [7, 8] that the conditional probability for the event A to happen when the event B has already happened is calculated by

$$
\mathrm{P}(\mathrm{A} / \mathrm{B})=\frac{\mathrm{P}(\mathrm{A} \cap \mathrm{B})}{\mathrm{P}(\mathrm{B})}
$$


In case of finite sample spaces ,for example, with equally probable singleton events, the mathematical definition of probability gives that $\mathrm{P}(\mathrm{A} / \mathrm{B})=\mathrm{N}_{\mathrm{A} \cap \mathrm{B}}: \mathrm{N}_{\mathrm{B}}$, where $N_{A \cap B}$ and $N_{B}$ denote the numbers of appearance of the events $\mathrm{A} \cap \mathrm{B}$ and $\mathrm{B}$ respectively .Therefore, if $\mathrm{N}$ is the cardinality of the sample space of $\mathrm{B}$, then $\mathrm{P}(\mathrm{A} / \mathrm{B})=\left(\mathrm{N}_{\mathrm{A} \cap \mathrm{B}}: \mathrm{N}\right):\left(\mathrm{N}_{\mathrm{B}}: \mathrm{N}\right)$, which proves (1).

In the same way one finds that

$$
\mathrm{P}(\mathrm{B} / \mathrm{A})=\frac{\mathrm{P}(\mathrm{A} \cap \mathrm{B})}{\mathrm{P}(\mathrm{A})} \text { or } \mathrm{P}(\mathrm{A} \cap \mathrm{B})=\mathrm{P}(\mathrm{B} / \mathrm{A}) \mathrm{P}(\mathrm{A}) \text {. }
$$

Therefore (1) can be written in the form

$$
\mathrm{P}(\mathrm{A} / \mathrm{B})=\frac{\mathrm{P}(\mathrm{B} / \mathrm{A}) \mathrm{P}(\mathrm{A})}{\mathrm{P}(\mathrm{B})}
$$

Equation (2), which calculates the conditional probability $\mathrm{P}(\mathrm{A} / \mathrm{B})$ with the help of the inverse in time conditional probability $\mathrm{P}(\mathrm{B} / \mathrm{A})$, the prior probability $\mathrm{P}(\mathrm{A}$ ) and the posterior probability $\mathrm{P}(\mathrm{B})$, is known as the Bayes' theorem (or rule, or law).

In other words, the Bayes' theorem calculates the probability of an event based on prior knowledge of conditions related to that event. However, when applied in practice, the Bayes' theorem may have several interpretations.

In social sciences, for example, it describes how a degree of belief expressed as a probability $\mathrm{P}(\mathrm{A})$ is rationally changed according to the availability of related evidence. In that case, the probabilities involved in the Bayes' theorem are frequently referred as Bayesian probabilities, although, mathematically speaking, Bayesian and conditional probabilities are actually the same thing.

The value of the prior probability $\mathrm{P}(\mathrm{A})$ is fixed before the experiment, whereas the value of the posterior probability is derived from the experiment's data. Usually, however, there exists an uncertainty about the exact value of $\mathrm{P}(\mathrm{A})$. In such cases, considering all the possible values of $\mathrm{P}(\mathrm{A})$, we obtain different values for the conditional probability $\mathrm{P}(\mathrm{A} / \mathrm{B})$. Therefore, the Bayes' rule introduces a kind of multivalued logic tackling the existing, due to the imprecision of the value of the prior probability, uncertainty. Consequently, one could argue that Bayesian Reasoning constitutes an interface between bivalent and fuzzy logic.
The Bayes' rule was first appeared in the work "An Essay towards a Problem in the Doctrine of Chances" of the $18^{\text {th }}$ century British mathematician and theologian Thomas Bayes (Figure 1).

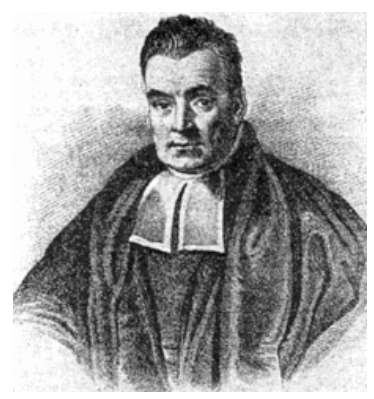

Figure 1: Thomas Bayes (1701-1761)

This essay was published by Richard Price in 1763, after the Bayes' death, in the "Philosophical Transactions of the Royal Society of London". The famous French mathematician Laplace (1749-1827), independently from Bayes, pioneered and popularized the Bayesian probabilities. The Bayes' theorem is frequently used together with the theorem of total probability [7] for the solution of more composite problems (e.g. see Example 5 of the next section).

In general, although the Bayes' rule is a simple consequence of the equation calculating the value of a conditional probability, Bayesian reasoning has been proved to be very important to everyday life situations [9] and for the whole science as well [10]. Recent researches give evidence that even the mechanisms under which the human brain works are Bayesian [11]! Consequently, Bayesian reasoning is very useful for Machine Learning, the sector of AI focusing on the design and construction of machines that mimic the human behavior. In fact, the smart machines of $\mathrm{AI}$ are supplied with Bayesian algorithms in order to be able to recognize the corresponding structures and to make autonomous decisions. The physicist and Nobel prize winner John Mather was one of the first who expressed his uneasiness about the possibility that the Bayesian machines could become too smart, so that to make humans to look useless [12]!

Sir Harold Jeffreys (1891-1989), a British mathematician who introduced the concept of the Bayesian algorithm and played an important role in the revival of the Bayesian view of probability, has successfully characterized the Bayesian rule 
as the "Pythagorean Theorem of Probability Theory" [13].

\section{Applications of Bayesian Reasoning to Everyday Life Situations}

Conditional probabilities and Bayesian reasoning have been proved very useful for solving problems appearing in everyday life situations. Some representative examples are presented in this section.

Example 1: A market's research is performed on the population of a town consisting $45 \%$ of men and $55 \%$ of women. Find the probability of the random choice of: i) Three men for the first three interviews, and ii) Four women for the next four interviews.

Solution: i) Let $A_{i}$ be the event that a man is chosen for the $i$-th interview, $i=1$, 2, 3. Then $\mathrm{P}\left(\mathrm{A}_{1}\right)=45: 100, \mathrm{P}\left(\mathrm{A}_{2} / \mathrm{A}_{1}\right)=$ 44:99 and $P\left(A_{3} / A_{1} \cap A_{2}\right)=43: 98$. Therefore, writing $\mathrm{P}\left(\mathrm{A}_{1} \cap \mathrm{A}_{2} \cap \mathrm{A}_{3}\right)=\mathrm{P}\left[\left(\mathrm{A}_{1} \cap \mathrm{A}_{2}\right) \cap \mathrm{A}_{3}\right]$ and applying two times equation (1) one finds that

$\mathrm{P}\left(\mathrm{A}_{1} \cap \mathrm{A}_{2} \cap \mathrm{A}_{3}\right)=\mathrm{P}\left(\mathrm{A}_{1} \cap \mathrm{A}_{2}\right) \mathrm{P}\left(\mathrm{A}_{3} / \mathrm{A}_{1} \cap \mathrm{A}_{2}\right)=$ $\mathrm{P}\left(\mathrm{A}_{1}\right) \mathrm{P}\left(\mathrm{A}_{2} / \mathrm{A}_{1}\right) \mathrm{P}\left(\mathrm{A}_{3} / \mathrm{A}_{1} \cap \mathrm{A}_{2}\right) \approx 0.088$ or $8.8 \%$.

ii) Given a finite number $n$ of events, one can show by induction that

$\mathrm{P}\left(\mathrm{A}_{1} \cap \mathrm{A}_{2} \cap \ldots . \cap \mathrm{A}_{\mathrm{n}}\right)=\mathrm{P}\left(\mathrm{A}_{1}\right) \mathrm{P}\left(\mathrm{A}_{2} / \mathrm{A}_{1}\right)$ $\mathrm{P}\left(\mathrm{A}_{3} / \mathrm{A}_{1} \cap \mathrm{A}_{2}\right) \quad \ldots \quad \mathrm{P}\left(\mathrm{A}_{\mathrm{n}} / \mathrm{A}_{1} \cap \mathrm{A}_{2} \cap \ldots \cap \mathrm{A}_{\mathrm{n}-1}\right)$ (3).

Let $A_{1}, A_{2}$ and $A_{3}$ be the events defined in case (i) and let $A_{i}$ be the event that a woman is chosen for the $\mathrm{i}$-th interview, $\mathrm{i}=$ 4, 5, 6, 7. Then

$\mathrm{P}\left(\mathrm{A}_{4} / \mathrm{A}_{1} \cap \mathrm{A}_{2} \cap \mathrm{A}_{3}\right)=55: 97 \approx 0.567$,

$\mathrm{P}\left(\mathrm{A}_{5} / \mathrm{A}_{1} \cap \mathrm{A}_{2} \cap \mathrm{A}_{3} \cap \mathrm{A}_{4}\right)=54: 96 \approx 0.562$,

$\mathrm{P}\left(\mathrm{A}_{6} / \mathrm{A}_{1} \cap \mathrm{A}_{2} \cap \mathrm{A}_{3} \cap \mathrm{A}_{4} \cap \mathrm{A}_{5}\right)=53: 95 \approx 0.558$, and $\mathrm{P}\left(\mathrm{A}_{7} / \mathrm{A}_{1} \cap \mathrm{A}_{2} \cap \mathrm{A}_{3} \cap \mathrm{A}_{4} \cap \mathrm{A}_{5} \cap \mathrm{A}_{6}\right)=52: 94$ $\approx 0.553$.

Therefore, applying equation ( 3 ) for $\mathrm{n}=7$ one finds that $\mathrm{P}\left(\mathrm{A}_{1} \cap \mathrm{A}_{2} \cap \ldots . \cap \mathrm{A}_{7}\right)=0.0086$ or $0.86 \%$.

Bayesian reasoning is frequently used in medical paradigms the outcomes of which are not always compatible to the common beliefs. The following three timely examples, due to the current COVID-19 pandemic, concern the creditability of the viruses' diagnostic tests.

Example 2: The statistical data show that $2 \%$ of the inhabitants of country have been infected by a dangerous virus. Mr. X, who has not any symptoms of the corresponding disease, makes a diagnostic test, the statistical accuracy of which is 97\%. The test is positive. What is the probability for Mr. X to be a carrier of the virus?

Solution: Consider the following events:

- A: The subject is a carrier of the virus.

- B: The test is positive.

On the basis of the given data it turns out that $\mathrm{P}(\mathrm{A})=0.02$ and $\mathrm{P}(\mathrm{B} / \mathrm{A})=0.97$.

Among 100 inhabitants of the country, 2 on average are carriers and 98 are noncarriers of the virus. Assuming that all those people make the test, we should have on average $2 \times 97 \%=1.94$ positive tests from the carriers and $98 \times 3 \%=2.94$ positive tests from the noncarriers of the virus, i.e.4.88 in total positive tests. Therefore, $\mathrm{P}(\mathrm{B})=0.488$. Replacing the values of $\mathrm{P}(\mathrm{A}), \mathrm{P}(\mathrm{B} / \mathrm{A})$ and $\mathrm{P}(\mathrm{B})$ in equation (2) one finds that $\mathrm{P}(\mathrm{A} / \mathrm{B}) \approx 0.398$. Therefore, the probability for Mr. X to be a carrier of the virus is only $39.8 \%$ and not $97 \%$, as it could be thought through a first, rough estimation!

This means that $\mathrm{Mr}$. $\mathrm{X}$ has to make a second test to see what really happens with his health condition. Further, if the second test is negative, a third test will be also required. At the same time, however, there is an urgent need for other people to make the test. This becomes evident by the next example.

Example 3: Assume that $\mathrm{Mr}$. $\mathrm{X}$ has some suspicious symptoms and that $85 \%$ of the people presenting such symptoms have been infected by the virus. Mr. X makes the test, which is positive. What is now the probability for $\mathrm{Mr}, \mathrm{X}$ to be a carrier of the virus?

Solution: Let A and B be the events defined in Example 2. Here we have that $\mathrm{P}(\mathrm{A})=0.85$ and $\mathrm{P}(\mathrm{B} / \mathrm{A})=0.97$. Further, assuming that 100 people having suspicious symptoms make the test, we should have on average $85 \times 97 \%=82.45$ positive tests from the carriers and $15 \times 0.3 \%=0.45$ from the noncarriers of the virus, i.e. 82.9 in total positive tests. Therefore, $\mathrm{P}(\mathrm{B})=0.829$.

Replacing the values of $\mathrm{P}(\mathrm{A}), \mathrm{P}(\mathrm{B} / \mathrm{A})$ and $\mathrm{P}(\mathrm{B})$ in equation (2) one finds that $\mathrm{P}(\mathrm{A} / \mathrm{B}) \approx 0.995$. In this case, therefore, the probability for Mr. X to be a carrier of the virus is $99.5 \%$, i.e. exceeds the statistical accuracy of the test!

In general, the sensitivity of the solution is great, depending on the values of the 
prior probability $\mathrm{P}(\mathrm{A})$. The greater the value of $\mathrm{P}(\mathrm{A})$, the higher the creditability of the test.

The next example examines what happens, if the test is negative.

Example 4: Assume that Mr. X makes a diagnostic test, which is negative. Find the probability to be a carrier of the virus:

i) Under the conditions of Example 2, and

ii) under the conditions of Example 3.

Solution: Consider the following events:

- A: The subject is a carrier of the virus.

- $\mathrm{B}$ : The test is negative.

i) In this case we have $\mathrm{P}(\mathrm{A})=0.02$ and $\mathrm{P}(\mathrm{B} / \mathrm{A})=0.03$. Assuming that 100 people make the test, we should have on average $98 \times 97 \%=95.06$ negative tests from the noncarriers and $2 \times 3 \%=0.06$ from the carriers of the virus, i.e. an average of 95.12 in total negative tests. Therefore, $\mathrm{P}(\mathrm{B})=0.9512$.

Replacing the values of $\mathrm{P}(\mathrm{A}), \mathrm{P}(\mathrm{B})$ and $\mathrm{P}(\mathrm{B} / \mathrm{A})$ to equation (1) one finds that $\mathrm{P}(\mathrm{A} / \mathrm{B}) \approx 0.0006$. Therefore, the probability for Mr. X to be a carrier of the virus is only $0.06 \%$.

ii) Here we have $\mathrm{P}(\mathrm{A})=0.85$ and $\mathrm{P}(\mathrm{B} / \mathrm{A})=0.03$. Further, assuming that 100 people make the test, we shall have on average $15 \times 97 \%=14.55$ negative tests from the noncarriers and $85 \times 3 \%=2.55$ from the carriers of the virus, i.e. an average of 17.1 in total negative tests. Therefore, $\mathrm{P}(\mathrm{B})=0.171$.

Replacing the values of $\mathrm{P}(\mathrm{A}), \mathrm{P}(\mathrm{B})$ and $\mathrm{P}(\mathrm{B} / \mathrm{A})$ to equation (1) one finds that $\mathrm{P}(\mathrm{A} / \mathrm{B}) \approx 0.1491$. Therefore, the probability for $\mathrm{Mr}$. $\mathrm{X}$ to be a carrier of the virus is $14.91 \%$. One observes here that the greater the value of the prior probability $\mathrm{P}(\mathrm{A})$, the lower the creditability of the test.

Remark: The outcomes of the previous three examples support the view of many epidemiologists that, at the initial stage, the "blind" diagnostic tests for COVID-19 performed on the general population are not effective, burdening purposeless the healthcare system of the corresponding country.

To check this from another optical angle, one has to take into account the statistical estimation that the existing diagnostic tests for COVID-19 give 30\% incorrectly negative (IN) results and $10 \%$ incorrectly positive (IP) results. Assume that $2 \%$ of the population of a country has been infected by the coronavirus of COVID-19 and that the government decides to undergo the heavy cost of performing one million "blind" tests on the general population.

Among those people, 20000 on average should be carriers and 980000 noncarriers of the virus. Therefore, we should have $20000 \times 30 \%=6000$ on average IN results and 14000 correctly positive (CP) results from the carriers and $980000 \times 10 \%=98000$ IP results from the noncarriers of the virus. This means that 6000 people infected by the virus with IN tests will not take the required precautions, therefore transmitting easily the virus to the other people.

Further, denote, for simplicity, by $\mathrm{CP}$ and IP the numbers of CP and IP results of the tests respectively. Then, the probability $\mathrm{P}(\mathrm{CP})$ of a positive test to be correct is equal to

$$
\begin{array}{ccc}
\mathrm{P}(\mathrm{CP})=\mathrm{CP}:(\mathrm{CP}+\mathrm{IP})(4) \\
\text { In } \quad \text { our } \quad \text { case, } & \mathrm{P}(\mathrm{CP})
\end{array}
$$
$=14000:(14000+98000) \approx 0.125$, i.e. only $12.5 \%$ ! Therefore, there is an urgent need for the 112000 in total people with positive tests to make a second test in order to check their real health condition, etc.

Equation (4) shows that $\mathrm{P}(\mathrm{CP})$ increases, either if the number $\mathrm{CP}$ increases or if the number IP decreases. The former happens if more people are infected by the virus, whereas the latter will happen if the quality of the diagnostic tests will be improved.

When, for example, $20 \%$ of the population is infected by the virus, it is straightforward to check that the probability $\mathrm{P}(\mathrm{CP})$ will be approximately equal to $63.6 \%$. Consequently, the more people are infected by the virus, the higher the creditability (and therefore the usefulness) of the diagnostic tests in detecting the positive cases.

Our last example concerns a combination of the Bayes' rule and the theorem of total probability for the solution of the corresponding problem

Example 5: A country consists of three confederate districts, say $\mathrm{D}_{1}, \mathrm{D}_{2}$ and $\mathrm{D}_{3}$, where it lives the $20 \%, 25 \%$ and $55 \%$ of its total population respectively. A percentage of $60 \%, 45 \%$ and $10 \%$ respectively of the population of each one of those districts is against the confederation wanting for its district to be an independent country. What is the probability that one of those people, chosen randomly, lives in district $\mathrm{D}_{3}$ ? 
Solution: Consider the events

- $\mathrm{A}_{\mathrm{i}}$ : A person lives in district $\mathrm{D}_{\mathrm{i}}, \mathrm{i}=1$, 2,3 , and

- B: A person is against the confederation

On the basis of the given data it turns out that $\mathrm{P}\left(\mathrm{A}_{1}\right)=0.2, \mathrm{P}\left(\mathrm{A}_{2}\right)=0.25, \mathrm{P}\left(\mathrm{A}_{3}\right)=0.55$ and $\mathrm{P}\left(\mathrm{B} / \mathrm{A}_{1}\right)=0.6, \mathrm{P}\left(\mathrm{B} / \mathrm{A}_{2}\right)=0.45, \mathrm{P}\left(\mathrm{B} / \mathrm{A}_{3}\right)=$ 0.1 . We want to calculate the probability

$$
\mathrm{P}\left(\mathrm{A}_{3} / \mathrm{B}\right)=\left[\mathrm{P}\left(\mathrm{B} / \mathrm{A}_{3}\right) \mathrm{P}\left(\mathrm{A}_{3}\right)\right]: \mathrm{P}(\mathrm{B})
$$

The $\mathrm{A}_{\mathrm{i}}$ 's are obviously pairwise disjoint events and their union is equal to the sample space $\mathrm{X}$ of the inhabitants of the country (mathematically speaking the $A_{i}$ 's form a partition of $\mathrm{X}$ ). Therefore, by the theorem of total probability [6] one finds that

$\mathrm{P}(\mathrm{B})=\mathrm{P}\left(\mathrm{A}_{1} \cap \mathrm{B}\right)+\mathrm{P}\left(\mathrm{A}_{2} \cap \mathrm{B}\right)+\mathrm{P}\left(\mathrm{A}_{3} \cap \mathrm{B}\right)$ and,

by the Bayes' theorem,

$$
\begin{aligned}
& \mathrm{P}(\mathrm{B})=\mathrm{P}\left(\mathrm{B} / \mathrm{A}_{1}\right) \mathrm{P}\left(\mathrm{A}_{1}\right)+\mathrm{P}\left(\mathrm{B} / \mathrm{A}_{2}\right) \mathrm{P}\left(\mathrm{A}_{2}\right)+ \\
& +\mathrm{P}\left(\mathrm{B} / \mathrm{A}_{3}\right) \mathrm{P}\left(\mathrm{A}_{3}\right)
\end{aligned}
$$

Replacing the values of the probabilities involved in equation (5) one finds that $\mathrm{P}(\mathrm{B})=0.2875$. Therefore, equation (4) gives that $\mathrm{P}\left(\mathrm{A}_{3} / \mathrm{B}\right) \approx 0.0628$ or $6.28 \%$

\section{4, Bayesian Reasoning in Science}

Many scientists and philosophers of science argue nowadays that the whole science could be considered as a Bayesian process [9-11]. In this section we are going to support and justify this view.

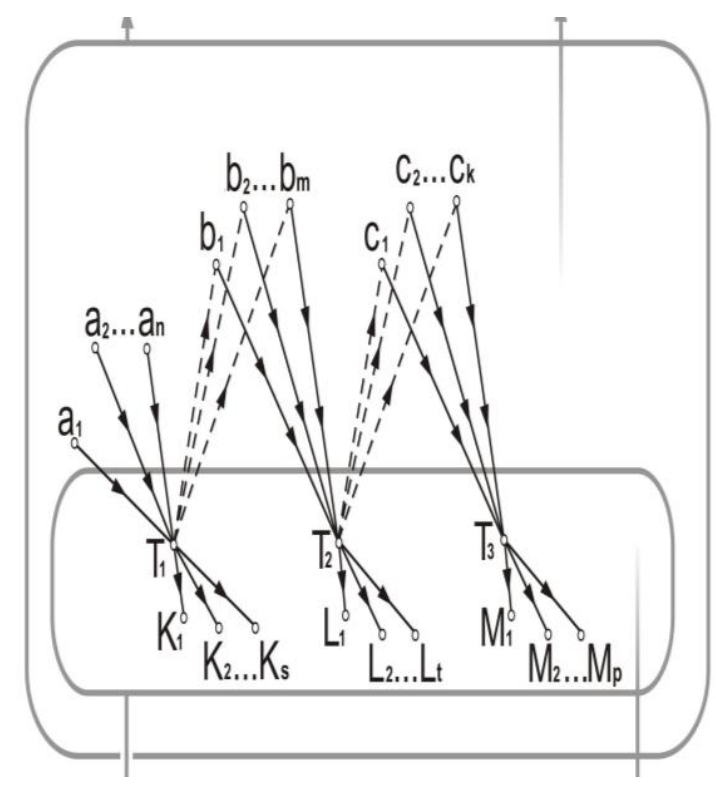

Figure 2: The scientific method

The process of scientific thinking, being a synthesis of inductive and deductive reasoning, is graphically represented in Figure 2, retrieved from [10]. The whole process for explaining a phenomenon starts with the humans' observations $a_{1}, a_{2}, \ldots, a_{n}$ of the real world connected to it, which laid by induction (intuitively) to the development of theory $\mathrm{T}_{1}$ about this phenomenon. Theory $\mathrm{T}_{1}$ is verified by deductive reasoning and additional deductive inferences $K_{1}, K_{2}, \ldots, K_{s}$ arre obtained. Next, a new series of observations $b_{1}, b_{2}, \ldots, b_{m}$ follow. If some of those observations are not compatible to the laws of theory $T_{1}$, a new theory $T_{2}$ is developed to replace/extend $T_{1}$, and so on. In each case the new theory extends or rejects the previous one approaching more and more to the objective truth related to the corresponding phenomenon.

This procedure is known as the scientific method. The term was introduced during the $19^{\text {th }}$ century, when significant terminologies appeared establishing clear boundaries between science and non science. However, the scientific method characterizes the development of science since at least the $17^{\text {th }}$ century. Aristotle (384-322 BC) is recognized as the inventor of the scientific method due to his refined analysis of the logical implications contained in demonstrative discourse. The first book in the history of human civilization written on the basis of the principles of the scientific method is, according to the existing witnesses, the "Elements" of Euclid (365300 BC) addressing the axiomatic foundation of Geometry.

The scientific method is highly based on the Trial and Error procedure, a term introduced by C. Lloyd Morgan (18521936) [14]. This procedure is characterized by repeated attempts, which are continued until success or until the subject stops trying.

As an example, the geocentic theory (Almagest) of Ptolemy of Alexandria (100170), being able to predict satisfactorily the movements of the planets and the moon, was considered to be true for centuries. However, it was finally proved to be wrong and has been replaced by the heliocentric theory of Copernicus (1473-1543). The Copernicus' theory was supported and enhanced a hundred years later by the observations/studies of Kepler and Galileo, but it faced many obstacles for a long 
period, especially from the church, before its final justification [15].

Another characteristic example is the Einstein's general relativity theory developed at the beginning of the $20^{\text {th }}$ century. This theory has replaced the Newton's classical gravitational theory, which was believed to be true for more than two centuries. The Einstein's new approach was based on the fact that, according to his special theory of relativity (1905) the distance $(\mathrm{r})$ and the time $(\mathrm{t})$ are changing in a different way with respect to a motionless and to a moving observer.

To support his argument Einstein introduced the concept of the 4-dimensional time-space and after a series of intensive efforts (1908-1915) he finally managed to prove that the geometry of this space is non Euclidean. This can be physically explained by the distortion created to the time-space due to the presence of mass or of an equivalent amount of energy, which looks analogous to the distortion created by a ball of bowling on the level of a trampoline.

Einstein's theory was experimentally verified by the irregularity of the Hermes' orbit around the sun and later by the magnitude of the light's divergence, which was calculated during the eclipse of the sun on May 29, 1919. In fact, the eclipse let some stars, which normally should be behind the sun, to appear besides it on the sky [16].

The previous discussion about the scientific method reveals the importance of inductive reasoning for scientific thinking. In fact, the premises of all the scientific theories (with possible exception only for pure mathematics), expressed by axioms, basic principles, etc., are based on human intuition and inductive reasoning. Therefore, a deductive inference developed on the basis of a scientific theory, is true under the CONDITION that the premises of the corresponding theory are true. In other words, if $\mathrm{H}$ denotes the hypothesis imposed by those premises and I denotes the deductive inference, then the conditional probability $\mathrm{P}(\mathrm{I} / \mathrm{H})$, which can be calculated by the Bayes' rule, expresses the degree of truth of the deductive inference. Consequently, the argument that the WHOLE SCIENCE is characterized by Bayesian reasoning seems to be true.

It must be emphasized that the error of the inductive reasoning is transferred to a deductive inference through its premises. Therefore, the scientific error in its final form is actually a deductive and not an inductive error! This means that none of the existing scientific theories could be considered as been absolutely true; it simply could be considered as approaching the truth in a better way than the previous theories, that has replaced, did.

\section{Conclusion}

In the present study was shown that Bayesian Reasoning could be considered as an interface between bivalent and fuzzy logic. Its usefulness to everyday life situations was also illustrated by suitable examples and its importance for the whole science was studied.

\section{References:}

[1] Moor, J. The Dartmouth College Artificial Intelligence conference: The next fifty years, AI Magazine, Vol. 27, 2006, pp.87-91.

[2] Hodges, A., Alan Turing: The Enigma, Centenary Edition, Princeton University Press: Princeton, NJ, USA, 2012.

[3] Janes, E.T., Probability Theory: The Logic of Science, Cambridge University Press, UK, $8^{\text {th }}$ Printing, 2011 (first published, 2003).

[4] Mumford, D., The Dawning of the Age of Stochasticity, in V. Amoid, M. Atiyah, P. Laxand \& B. Mazur (Eds.), Mathematics: Frontiers and Perspectives, AMS, 197-218, 2000.

[5] Kosko, B. Fuzzy Thinking: The New Science of Fuzzy Logic, Hyperion New York 1993

[6] Shahbazova, S.N., Sugeno, M., Kacpzyk, J. (Eds.), Recent Developments in Fuzzy Logic and Fuzzy Sets (dedicated to L.A.Zadeh), Spinger,NY,2020.

[7] Schuler, J. \& Lipschutz, S., Schaum's Outline of Probability, $2^{\text {nd }}$ Edition, McGraw-Hill, NY, USA, 2010.

[8] Shiryaev, A.N., Probability-1, 3d Edition, Spinger,NY,2016.

[9] Horgan, J., Bayes' Theorem: What is the Big Deal?", January 2015, available in http//:blogs. scientificamerican.com /cross-check/ bayes-s-theorem-what-s-the-big-deal.

[10] Athanassopoulos, E. and Voskoglou, M.Gr., A Philosophical Treatise on the 
Connection of Scientific Reasoning with Fuzzy Logic, Mathematics, 8, article 875, 2020.

[11] Bertsch McGrayne, S., The Theory that would not die, Yale University Press, New Haven and London, 2012.

[12] What do you think about machines that think? 2015, available in http://edge.org/response-detail/26871

[13] Jeffreys, H., Scientific Inference, 3d Edition, Cambridge University Press, UK, 1973.

[14] Thrope, W.H., The origins and rise of ethology: The science of the natural behavior of animals, Praeger, LondonNY, 1979.

[15] Gingerich, O., The Eye of the HeavenPtolemy, Copernicus, Kepler, American Institute of Physics, NY, 1993.

[16] Singh, S., Bing Bang - The Origin of the Universe, Harper Perennian Publishers, NY, 2005.

\section{Creative Commons Attribution License 4.0 (Attribution 4.0 International, CC BY 4.0)}

This article is published under the terms of the Creative Commons Attribution License 4.0

https://creativecommons.org/licenses/by/4.0/deed.en_US 